\title{
Some dynamic inequalities on time scales and their applications
}

\author{
A.A. El-Deeb ${ }^{1 *}$, Haiyong $\mathrm{Xu}^{2}$, A. Abdeldaim ${ }^{3,4}$ and Guotao Wang ${ }^{5,6,7}$
}

"Correspondence:

ahmedeldeeb@azhar.edu.eg

'Department of Mathematics,

Faculty of Science, Al-Azhar

University, Cairo, Egypt

Full list of author information is

available at the end of the article

\begin{abstract}
This paper is devoted to the study of Gronwall-Bellman-type inequalities on an arbitrary time scale $\mathbb{T}$. We investigate some new explicit bounds of a certain class of nonlinear retarded dynamic inequalities of Gronwall-Bellman type on time scales. These inequalities extend some known dynamic inequalities on time scales. We also generalize and unify some continuous inequalities and their corresponding discrete analogues. To illustrate the benefits of our work, we present some applications of these results. The main results will be proved by using some analysis techniques and a simple consequence of the Keller's chain rule on time scales.
\end{abstract}

MSC: 26D10; 26D15; 26D20; 34A12; 34A40

Keywords: Dynamic inequalities of Gronwall-Bellman type; Analysis techniques; Keller's chain rule on time scales

\section{Introduction}

In 1919, Gronwall [1] discovered a celebrated inequality. He proved that if $\varpi$ is a continuous function defined on the interval $D=[a, a+h]$ and

$$
0 \leq \varpi(t) \leq \int_{a}^{t}[\zeta \varpi(s)+\xi] d s, \quad t \in D,
$$

where $a, \xi, \zeta$, and $h$ are nonnegative constants, then

$$
0 \leq \varpi(t) \leq \xi h e^{\zeta h}, \quad t \in D .
$$

This inequality has been very important in the theory of differential equations and difference equations. After that, in 1943, Bellman [2] proved a fundamental inequality, as a generalization for Gronwall's inequality (1.1): If $\varpi$ and $f$ are continuous nonnegative functions defined on $[a, b]$, and $c$ is a nonnegative constant, then the inequality

$$
\varpi(t) \leq c+\int_{a}^{t} f(s) \varpi(s) d s, \quad t \in[a, b],
$$

implies that

$$
\varpi(t) \leq c \exp \left(\int_{a}^{t} f(s) d s\right), \quad t \in[a, b] .
$$

(c) The Author(s) 2019. This article is distributed under the terms of the Creative Commons Attribution 4.0 International License (http://creativecommons.org/licenses/by/4.0/), which permits unrestricted use, distribution, and reproduction in any medium, provided you give appropriate credit to the original author(s) and the source, provide a link to the Creative Commons license, and indicate if changes were made. 
Bellman [2] gave a generalization of (1.2). He proved that if $\varpi, f, a, \in C\left(\mathbb{R}_{+}, \mathbb{R}_{+}\right)$and $a$ is a nondecreasing function, then the inequality

$$
\varpi(t) \leq a(t)+\int_{0}^{t} f(s) \varpi(s) d s, \quad t \in \mathbb{R}_{+},
$$

implies

$$
\varpi(t) \leq a(t) \exp \left(\int_{0}^{t} f(s) d s\right), \quad t \in \mathbb{R}_{+} .
$$

The celebrated Gronwall-Bellman inequality [3] in its discrete form asserts that if $\varpi(n)$, $a(n)$, and $b(n)$ are nonnegative sequences defined for $n \in \mathbb{N}_{0}$ and $a(n)$ is a nondecreasing sequence for $n \in \mathbb{N}_{0}$ and if

$$
\varpi(n) \leq a(n)+\sum_{s=0}^{n-1} f(n) \varpi(n), \quad n \in \mathbb{N}_{0}
$$

then

$$
\varpi(n) \leq a(n) \prod_{s=0}^{n-1}[1+f(n)], \quad n \in \mathbb{N}_{0} .
$$

Gronwall-Bellman-type inequalities and their generalizations have received much attention from many authors (see [4-9]). For example, Pachpatte [8] established the following further generalization of the Gronwall-Bellman inequality:

$$
\varpi(t) \leq \varpi_{0}+\int_{0}^{t}[f(s) \varpi(s)+p(s)] d s+\int_{0}^{t} f(s)\left(\int_{0}^{s} g(\sigma) \varpi(\sigma) d \sigma\right) d s
$$

for $t \in(0, \infty)$.

Ferreira et al. [5] established the following inequality:

$$
\phi(\varpi(t)) \leq c(t)+\int_{0}^{t}\left[f(t, s) \omega_{1}(\varpi(s)) \omega_{2}(\varpi(s))+g(t, s) \omega_{2}(\varpi(s))\right] d s, \quad t \in \mathbb{R}^{+} .
$$

Retarded integral inequalities play an important role in the study of various types of retarded differential and integral equations (see [10-13]). El-Owaidy, Abdeldaim, and ElDeeb [13] established new retarded nonlinear integral inequalities

$$
\varpi(t) \leq b(t)+\int_{a}^{t} g(s) \varpi^{p}(s) d s+\int_{a}^{\alpha(t)} h(s) \varpi^{p}(s) d s
$$

and

$$
\varpi^{p}(t) \leq b^{p}(t)+\int_{a}^{t} g(s) \varpi^{p}(s) d s+\int_{a}^{\alpha(t)} h(s) \varpi^{q}(s) d s .
$$

During the past ten years, many authors have studied several inequalitiesto achieve several objects (see $[8,11,13-19]$, and the references therein). 
As a unification of the continuous version inequality (1.3) and the discrete version inequality (1.4), Bohner and Peterson [20] proved that if $\chi$ and $\delta$ are right-dense continuous functions and $\gamma \geq 0$ is a regressive right-dense continuous function, then

$$
\varpi(t) \leq \delta(t)+\int_{t_{0}}^{t} \varpi(\eta) \gamma(s) \Delta \eta, \quad t \in \mathbb{T},
$$

implies

$$
\varpi(t) \leq \delta(t)+\int_{t_{0}}^{t} e_{\gamma}(t, \sigma(\eta)) \delta(\eta) \gamma(s) \Delta \eta, \quad t \in \mathbb{T},
$$

where $\mathbb{T}$ is an arbitrary closed subset of the real numbers $\mathbb{R}$ which is called a time scale. Stefan Hilger is the first to discover the theory of time scales in his $\mathrm{PhD}$ thesis [21]. For more information and details on the time scales, we refer the reader to the books [22, 23]. Many dynamic inequalities have been studied by different authors during the past decade (see [20, 24-36] and the references therein). Throughout this paper, the knowledge and understanding of the time scales and time scale notion are assumed.

Li [31] extended inequality (1.5) to time scales as follows:

$$
\varpi(t) \leq \varpi_{0}+\int_{0}^{t}[f(s) \varpi(s)+p(s)] \Delta s+\int_{0}^{t} f(s)\left(\int_{0}^{s} g(\sigma) \varpi(\sigma) \Delta \sigma\right) \Delta s .
$$

Feng et al. [37] generalized inequality (1.6) to time scales as follows:

$$
\begin{aligned}
\phi(\varpi(t)) \leq & c(t)+\int_{0}^{t}\left[f(t, s) \omega_{1}(\varpi(s)) \omega_{2}(\varpi(s))+g(t, s) \omega_{2}(\varpi(s))\right] \Delta s \\
& +\int_{t_{0}}^{t} \int_{t_{0}}^{s} h(\delta) \omega_{1}(\varpi(\delta)) \Delta \delta \Delta s .
\end{aligned}
$$

In the following, we present the basic lemmas needed in the proof of our main results.

Lemma 1.1 (See [31]) If $\pi \in \Re$ and $t \in \mathbb{T}$, then the exponential function $e_{\pi}\left(t, t_{0}\right)$ is a unique solution of the following initial value problem:

$$
\left\{\begin{array}{l}
\varphi^{\Delta}(t)=\pi(t) \varphi(t), \\
\varphi\left(t_{0}\right)=1 .
\end{array}\right.
$$

Lemma 1.2 (See [31]) Let $t_{0} \in \mathbb{T}^{\kappa}$ and $\varsigma: \mathbb{T} \times \mathbb{T}^{\kappa} \rightarrow \mathbb{R}$ be continuous at $(t, t)$, where $t>t_{0}$ and $t \in \mathbb{T}^{\kappa}$. Assume that $\varsigma^{\Delta}(t, \cdot)$ is $r d$-continuous on $\left[t_{0}, \sigma(t)\right]_{\mathbb{T}}$. Suppose that, for any $\varepsilon>0$, there exists a neighborhood $U$ of $t$, independent of $\lambda \in\left[t_{0}, \sigma(t)\right]_{\mathbb{T}}$, such that

$$
\left|[\varsigma(\sigma(t), \lambda)-\varsigma(s, \lambda)]-\varsigma^{\Delta}(t, \lambda)[\sigma(t)-s]\right| \leq \varepsilon|\sigma(t)-s|, \quad s \in U,
$$

where $\varsigma^{\Delta}$ denotes the derivative of $\varsigma$ with respect to the first variable. Then

$$
\varpi(t)=\int_{t_{0}}^{t} \varsigma(t, \lambda) \Delta \lambda
$$


yields

$$
\varpi^{\Delta}(t)=\int_{t_{0}}^{t} \varsigma^{\Delta}(t, \lambda) \Delta \lambda+\varsigma(\sigma(t), t) .
$$

Lemma 1.3 (See [20, p. 28, Thm. 1.76]) If $\varpi^{\Delta}(t) \geq 0$, then $\varpi(t)$ is nondecreasing.

Lemma 1.4 (See [20, p. 32, Thm. 1.90]. Chain rule) Let $\varpi: \mathbb{R} \rightarrow \mathbb{R}$ be continuously differentiable and suppose $\varrho: \mathbb{T} \rightarrow \mathbb{R}$ is delta differentiable. Then $\varpi \circ \varrho: \mathbb{T} \rightarrow \mathbb{R}$ is delta differentiable, and

$$
(\varpi \circ \varrho)^{\Delta}(t)=\left\{\int_{0}^{1}\left[\varpi^{\prime}\left(\varrho(t)+h \mu(t) \varrho^{\Delta}(t)\right)\right] d h\right\} \varrho^{\Delta}(t) .
$$

Lemma 1.5 (See [20, p. 9, Exercise 1.23]) Assume that $\varpi: \mathbb{T} \rightarrow \mathbb{R}$ is a delta differentiable function at $x \in \mathbb{T}^{\kappa}$. Then

$$
\left(\varpi^{n}\right)^{\Delta}(x)=\left\{\sum_{k=0}^{n-1} \varpi^{k}(x)\left[\varpi^{\sigma}(x)\right]^{n-1-k}\right\} \varpi^{\Delta}(x) .
$$

Lemma 1.6 (See $[20$, p. 5, Thm. 1.16]) Assume that $\varpi: \mathbb{T} \rightarrow \mathbb{R}$ is a delta differentiable function at $x \in \mathbb{T}^{\kappa}$. Then

$$
\varpi^{\sigma}(x)=\varpi(x)+\mu(x) \varpi^{\Delta}(x) .
$$

Lemma 1.7 (See [38, p. 423, Lemma 2.5]) Let $a, b \in \mathbb{T}$ and $p>1$. Assume that $\varpi: \mathbb{T} \rightarrow \mathbb{R}$ is delta differentiable at $x \in \mathbb{T}^{\kappa}$ and nonnegative increasing function on $[a, b]_{\mathbb{T}}$. Then

$$
p \varpi^{p-1}(x) \varpi^{\Delta}(x) \leq\left(\varpi^{p}\right)^{\Delta}(x) \leq p\left(\varpi^{\sigma}(x)\right)^{p-1} \varpi^{\Delta}(x) .
$$

In this paper, we investigate some delay dynamic integral inequalities on time scales, which extend some known retarded dynamic inequalities on time scales, also unify and extend some continuous inequalities and their corresponding discrete analogues in the literature. The paper is organized as follows: In Sect. 2, we state and prove the main results. In Sect. 3, we present several applications to study some qualitative properties of the solutions of certain retarded dynamic equations.

\section{Main results}

This section states and proves our results. Throughout this paper, we always assume that $t_{0} \in \mathbb{T}$ and $\mathbb{T}_{0}=\left[t_{0}, \infty\right) \cap \mathbb{T}$.

Theorem 2.1 Suppose that $u, a \in C_{\mathrm{rd}}\left(\mathbb{T}_{0}, \mathbb{R}^{+}\right)$, a is nondecreasing, $g(t, s), g^{\Delta_{t}}(t, s), h(t, s)$, $h^{\Delta_{t}}(t, s) \in C_{\mathrm{rd}}\left(\mathbb{T}_{0} \times \mathbb{T}_{0}, \mathbb{R}^{+}\right), \omega_{1}, \omega_{2} \in C\left(\mathbb{R}^{+}, \mathbb{R}^{+}\right)$are nondecreasing, $\tau(t) \in\left(\mathbb{T}_{0}, \mathbb{T}\right)$ with $\tau(t)<$ $t,-\infty<\alpha=\inf \left\{\tau(t), t \in \mathbb{T}_{0}\right\} \leq t_{0}$, and $\phi \in C_{\mathrm{rd}}\left(\left[\alpha, t_{0}\right] \cap \mathbb{T}, \mathbb{R}^{+}\right)$. If for $t \in \mathbb{T}_{0}$, u satisfies the inequality:

$$
\omega_{1}(u(t)) \leq a(t)+\int_{t_{0}}^{t} g(t, s) \omega_{1}(u(\tau(s))) \Delta s+\int_{t_{0}}^{t} h(t, s) \omega_{2}(u(\tau(s))) \Delta s
$$


with the initial condition

$$
\left\{\begin{array}{l}
\omega_{1}(u(t))=\phi(t) \quad \text { if } t \in\left[\alpha, t_{0}\right] \cap \mathbb{T} \\
\phi(\tau(t)) \leq a(t) \quad \text { if } \tau(t) \leq t_{0}, t \in \mathbb{T}_{0}
\end{array}\right.
$$

then

$$
u(t) \leq \omega_{1}^{-1}\left\{G^{-1}\left[H^{-1}\left\{H\left(G(a(t))+\int_{t_{0}}^{t} h(t, s) \Delta s\right)+\int_{t_{0}}^{t} g(t, s) \Delta s\right\}\right]\right\}
$$

with

$$
H\left(G(a(t))+\int_{t_{0}}^{t} h(t, s) \Delta s\right)+\int_{t_{0}}^{t} g(t, s) \Delta s \in \operatorname{Dom}\left(H^{-1}\right),
$$

where $G, H$ are increasing bijective functions defined by

$$
\begin{aligned}
(G \circ v)^{\Delta}(t) & =\frac{v^{\Delta}(t)}{\omega_{2}\left(\omega_{1}^{-1}(v(t))\right)}, \\
(H \circ z)^{\Delta}(t) & =\frac{\omega_{2}\left(\omega_{1}^{-1}\left(G^{-1}(z(t))\right)\right) z^{\Delta}(t)}{G^{-1}(z(t))} .
\end{aligned}
$$

Proof Fixing an arbitrary number $T^{*} \in \mathbb{T}_{0}$, for $t \in\left[t_{0}, T^{*}\right] \cap \mathbb{T}$, we define the function

$$
v(t)=a\left(T^{*}\right)+\int_{t_{0}}^{t} g(t, s) \omega_{1}(u(\tau(s))) \Delta s+\int_{t_{0}}^{t} h(t, s) \omega_{2}(u(\tau(s))) \Delta s .
$$

Clearly, $v$ is a nonnegative nondecreasing function, and we have

$$
u(t) \leq \omega_{1}^{-1}(v(t)), \quad t \in\left[t_{0}, T^{*}\right] \cap \mathbb{T} .
$$

Therefore, for $t \in\left[t_{0}, T^{*}\right] \cap \mathbb{T}$, if $\tau(t) \geq t_{0}$, then $\tau(t) \in\left[t_{0}, T^{*}\right] \cap \mathbb{T}$, and from (2.7) we obtain

$$
u(\tau(t)) \leq \omega_{1}^{-1}(v(\tau(t))) \leq \omega_{1}^{-1}(v(t)) .
$$

On the other hand, if $\tau(t) \leq t_{0}$, then, using the initial condition (2.2), we get

$$
u(\tau(t))=\phi(\tau(t)) \leq a(t) \leq \omega_{1}^{-1}(v(t)) .
$$

So, combining (2.8) and (2.9), we have

$$
u(\tau(t)) \leq \omega_{1}^{-1}(v(t)) .
$$

Moreover, from (2.6), Lemma 1.2, and (2.10) we get

$$
\begin{aligned}
v^{\Delta}(t)= & \int_{t_{0}}^{t} g^{\Delta_{t}}(t, s) \omega_{1}(u(\tau(s))) \Delta s+g(\sigma(t), t) \omega_{1}(u(\tau(t))) \\
& +\int_{t_{0}}^{t} h^{\Delta_{t}}(t, s) \omega_{2}(u(\tau(s))) \Delta s+h(\sigma(t), t) \omega_{2}(u(\tau(t)))
\end{aligned}
$$




$$
\begin{aligned}
\leq & \int_{t_{0}}^{t} g^{\Delta_{t}}(t, s) v(s) \Delta s+g(\sigma(t), t) v(t) \\
& +\int_{t_{0}}^{t} h^{\Delta_{t}}(t, s) \omega_{2}\left(\omega_{1}^{-1}(v(t))\right) \Delta s+h(\sigma(t), t) \omega_{2}\left(\omega_{1}^{-1}(v(s))\right) \\
\leq & \left\{\int_{t_{0}}^{t} g^{\Delta_{t}}(t, s) \Delta s+g(\sigma(t), t)\right\} v(t) \\
& +\left\{\int_{t_{0}}^{t} h^{\Delta_{t}}(t, s) \Delta s+h(\sigma(t), t)\right\} \omega_{2}\left(\omega_{1}^{-1}(v(t))\right),
\end{aligned}
$$

which implies

$$
\frac{v^{\Delta}(t)}{\omega_{2}\left(\omega_{1}^{-1}(v(t))\right)} \leq\left\{\int_{t_{0}}^{t} g(t, s) \Delta s\right\}^{\Delta_{t}} \frac{v(t)}{\omega_{2}\left(\omega_{1}^{-1}(v(t))\right)}+\left\{\int_{t_{0}}^{t} h(t, s) \Delta s\right\}^{\Delta_{t}} .
$$

Integrating (2.12) from $t_{0}$ to $t$ yields

$$
G(v(t))-G\left(v\left(t_{0}\right)\right) \leq \int_{t_{0}}^{t}\left\{\int_{t_{0}}^{s} g(s, \lambda) \Delta \lambda\right\}^{\Delta_{s}} \frac{v(s)}{\omega_{2}\left(\omega_{1}^{-1}(v(s))\right)} \Delta s+\int_{t_{0}}^{t} h(t, s) \Delta s .
$$

Since $v\left(t_{0}\right)=a\left(T^{*}\right)$ and $G$ is increasing, from (2.13) we have

$$
\begin{aligned}
v(t) \leq & G^{-1}\left[G\left(a\left(T^{*}\right)\right)+\int_{t_{0}}^{t}\left\{\int_{t_{0}}^{s} g(s, \lambda) \Delta \lambda\right\}^{\Delta_{s}} \frac{v(s)}{\omega_{2}\left(\omega_{1}^{-1}(v(s))\right)} \Delta s+\int_{t_{0}}^{t} h(t, s) \Delta s\right] \\
\leq & G^{-1}\left[G\left(a\left(T^{*}\right)\right)+\int_{t_{0}}^{t}\left\{\int_{t_{0}}^{s} g(s, \lambda) \Delta \lambda\right\}^{\Delta_{s}} \frac{v(s)}{\omega_{2}\left(\omega_{1}^{-1}(v(s))\right)} \Delta s\right. \\
& \left.+\int_{t_{0}}^{T^{*}} h(t, s) \Delta s\right] .
\end{aligned}
$$

For brevity, let

$$
z(t)=G\left(a\left(T^{*}\right)\right)+\int_{t_{0}}^{t}\left\{\int_{t_{0}}^{s} g(s, \lambda) \Delta \lambda\right\}^{\Delta_{s}} \frac{v(s)}{\omega_{2}\left(\omega_{1}^{-1}(v(s))\right)} \Delta s+\int_{t_{0}}^{T^{*}} h(t, s) \Delta s,
$$

and hence

$$
z\left(t_{0}\right)=G\left(a\left(T^{*}\right)\right)+\int_{t_{0}}^{T^{*}} h(t, s) \Delta s
$$

Then

$$
v(t) \leq G^{-1}(z(t)), \quad t \in\left[t_{0}, T^{*}\right] \cap \mathbb{T} .
$$

Using Lemma 1.2 and (2.15), we obtain

$$
\begin{aligned}
z^{\Delta}(t) & =\left\{\int_{t_{0}}^{t} g(t, s) \Delta s\right\}^{\Delta_{t}} \frac{v(t)}{\omega_{2}\left(\omega_{1}^{-1}(v(t))\right)} \\
& \leq\left\{\int_{t_{0}}^{t} g(t, s) \Delta s\right\}^{\Delta_{t}} \frac{G^{-1}(z(t))}{\omega_{2}\left(\omega_{1}^{-1}\left(G^{-1}(z(t))\right)\right)}
\end{aligned}
$$


which implies

$$
\frac{\omega_{2}\left(\omega_{1}^{-1}\left(G^{-1} z(t)\right)\right) z^{\Delta}(t)}{G^{-1}(z(t))} \leq\left\{\int_{t_{0}}^{t} g(t, s) \Delta s\right\}^{\Delta t} .
$$

Integrating (2.18) from $t_{0}$ to $t$ yields

$$
H(z(t))-H\left(z\left(t_{0}\right)\right) \leq \int_{t_{0}}^{t} g(t, s) \Delta s
$$

from which it follows that

$$
\begin{aligned}
z(t) & \leq H^{-1}\left\{H\left(z\left(t_{0}\right)\right)+\int_{t_{0}}^{t} g(t, s) \Delta s\right\} \\
& =H^{-1}\left\{H\left(G\left(a\left(T^{*}\right)\right)+\int_{t_{0}}^{T^{*}} h(t, s) \Delta s\right)+\int_{t_{0}}^{t} g(t, s) \Delta s\right\} .
\end{aligned}
$$

From (2.9), (2.16), and (2.19) we obtain

$$
u(t) \leq \omega_{1}^{-1}\left\{G^{-1}\left[H^{-1}\left\{H\left(G\left(a\left(T^{*}\right)\right)+\int_{t_{0}}^{T^{*}} h(t, s) \Delta s\right)+\int_{t_{0}}^{t} g(t, s) \Delta s\right\}\right]\right\} .
$$

Taking $t=T^{*}$ in (2.20) yields

$$
u\left(T^{*}\right) \leq \omega_{1}^{-1}\left\{G^{-1}\left[H^{-1}\left\{H\left[G\left(a\left(T^{*}\right)\right)+\int_{t_{0}}^{T^{*}} h(t, s) \Delta s\right]+\int_{t_{0}}^{T^{*}} g(t, s) \Delta s\right\}\right]\right\} .
$$

Since $T^{*} \in \mathbb{T}_{0}$ is chosen arbitrarily, we obtain the desired inequality after substituting $T^{*}$ with $t$ into (2.21). This completes the proof.

Remark 2.2 It is interesting to note that, in particular, if we put $\omega_{1}(u)=u^{p}$ and $g(t, s)=0$ in Theorem 2.1, then Theorem 2.1 reduces to [37, Thm. 3.1]. If also $\omega_{1}(u)=u^{p}, g(t, s)=0$, and $h(t, s)=b(t) f(s)$ in Theorem 2.1, then Theorem 2.1 reduces to [37, Thm. 3.2].

Theorem 2.1 unifies some known continuous and discrete inequalities in the literature as shown in the following remarks.

Remark 2.3 If we put $\mathbb{T}=\mathbb{R}, \omega_{1}(u)=u^{p}, \omega_{2}(u)=u^{q}, g(t, s)=f(s), h(t, s)=h(s), t_{0}=0$, and $a(t)=u_{0}$ (any constant) in Theorem 2.1, then Theorem 2.1 reduces to [7, Thm. 3.1].

Remark 2.4 If we put $\mathbb{T}=\mathbb{R}, \omega_{1}(u)=u, g(t, s)=0, t_{0}=0$ in Theorem 2.1, then Theorem 2.1 reduces to [39, Thm. 2.1]. If furthermore $h(t, s)=f_{1}(t) f_{2}(s)$ in Theorem 2.1, then Theorem 2.1 reduces to [39, Thm. 2.2].

Remark 2.5 If we take $\mathbb{T}=\mathbb{R}, \omega_{1}(u)=u^{p}, g(t, s)=0, t_{0}=0, \tau(t)=t$, and $h(t, s)=b(t) f(s)$ in Theorem 2.1, then Theorem 2.1 reduces to [8, Thm. 2(b3)]. If $\mathbb{T}=\mathbb{Z}, \omega_{1}(u)=u^{p}, g(t, s)=$ $0, t_{0}=0, \tau(t)=t$, and $h(t, s)=b(t) f(s)$ in Theorem 2.1, then Theorem 2.1 reduces to [8, Thm. $\left.4\left(d_{3}\right)\right]$. 
Theorem 2.6 Let $u, a, \tau, \omega_{1}, \omega_{2}, g$ be the same as in Theorem 2.1 , and let $f, p \in C_{\mathrm{rd}}\left(\mathbb{T}_{0}, \mathbb{R}^{+}\right)$. If $t \in \mathbb{T}_{0}$ and $u$ satisfies the inequality

$$
\begin{aligned}
\omega_{1}(u(t)) \leq & a(t)+\int_{t_{0}}^{t} \omega_{2}(u(\tau(s)))\left[f(s) \omega_{1}(u(\tau(s)))+p(s)\right] \Delta s \\
& +\int_{t_{0}}^{t} \omega_{2}(u(\tau(s))) f(s)\left[\int_{t_{0}}^{s} g(s, \lambda) \omega_{1}(u(\tau(\tau(\lambda)))) \Delta \lambda\right] \Delta s,
\end{aligned}
$$

then

$$
\begin{aligned}
u(t) \leq & \omega_{1}^{-1}\left\{\tilde { G } ^ { - 1 } \left[\tilde { H } ^ { - 1 } \left\{\tilde{H}\left[G(a(t))+\int_{t_{0}}^{t} p(s) \Delta s\right]\right.\right.\right. \\
& \left.\left.\left.+\int_{t_{0}}^{t} f(s)\left[1+\int_{t_{0}}^{s} g(s, \lambda) \Delta \lambda\right] \Delta s\right\}\right]\right\}
\end{aligned}
$$

with

$$
\tilde{H}\left[G(a(t))+\int_{t_{0}}^{t} p(s) \Delta s\right]+\int_{t_{0}}^{t} f(s)\left[1+\int_{t_{0}}^{s} g(s, \lambda) \Delta \lambda\right] \Delta s \in \operatorname{Dom}\left(\tilde{H}^{-1}\right),
$$

where $\tilde{G}$ and $\tilde{H}$ are increasing bijective functions defined by

$$
\begin{aligned}
& (\tilde{G} \circ v)^{\Delta}(t)=\frac{v^{\Delta}(t)}{\omega_{2}\left(\omega_{1}^{-1}(v(t))\right)}, \\
& (\tilde{H} \circ z)^{\Delta}(t)=\frac{z^{\Delta}(t)}{\tilde{G}^{-1}(z(t))} .
\end{aligned}
$$

Proof Fixing an arbitrary number $T^{*} \in \mathbb{T}_{0}$, for $t \in\left[t_{0}, T^{*}\right] \cap \mathbb{T}$, we define the function

$$
\begin{aligned}
v(t)= & a\left(T^{*}\right)+\int_{t_{0}}^{t} \omega_{2}(u(\tau(s)))\left[f(s) \omega_{1}(u(\tau(s)))+p(s)\right] \Delta s \\
& +\int_{t_{0}}^{t} \omega_{2}(u(\tau(s))) f(s)\left[\int_{t_{0}}^{s} g(s, \lambda) \omega_{1}(u(\tau(\tau(\lambda)))) \Delta \lambda\right] \Delta s .
\end{aligned}
$$

Clearly, $v$ is a nonnegative nondecreasing function with $v\left(t_{0}\right)=a\left(T^{*}\right)$, and we have

$$
u(t) \leq \omega_{1}^{-1}(v(t)), \quad t \in\left[t_{0}, T^{*}\right] \cap \mathbb{T} .
$$

Similarly to (2.8)-(2.9), we get

$$
u(\tau(t)) \leq \omega_{1}^{-1}(v(t)) .
$$

Furthermore, from (2.26), by Lemma 1.2 and (2.28) we have

$$
\begin{aligned}
v^{\Delta}(t)= & \omega_{2}(u(\tau(t)))\left[f(t) \omega_{1}(u(\tau(t)))+p(t)\right] \\
& +\omega_{2}(u(\tau(t))) f(t)\left[\int_{t_{0}}^{t} g(t, s) \omega_{1}(u(\tau(s))) \Delta s\right] \\
\leq & p(t) \omega_{2}\left(\omega_{1}^{-1}(v(t))\right)+f(t) \omega_{2}\left(\omega_{1}^{-1}(v(t))\right)\left[v(t)+\int_{t_{0}}^{t} g(t, s) v(s) \Delta s\right],
\end{aligned}
$$


El-Deeb et al. Advances in Difference Equations

(2019) 2019:130

Page 9 of 19

which implies

$$
\frac{v^{\Delta}(t)}{\omega_{2}\left(\omega_{1}^{-1}(v(t))\right)} \leq p(t)+f(t)\left[v(t)+\int_{t_{0}}^{t} g(t, s) v(s) \Delta s\right] .
$$

Integrating both sides of (2.30) from $t_{0}$ to $t$ yields

$$
\tilde{G}(v(t))-\tilde{G}\left(v\left(t_{0}\right)\right) \leq \int_{t_{0}}^{t} p(s) \Delta s+\int_{t_{0}}^{t} f(s)\left[v(s)+\int_{t_{0}}^{s} g(s, \lambda) v(\lambda) \Delta \lambda\right] \Delta s .
$$

Since $v\left(t_{0}\right)=a\left(T^{*}\right)$ and $\tilde{G}$ is an increasing function, from (2.31), we get

$$
\begin{aligned}
v(t) & \leq \tilde{G}^{-1}\left[G\left(a\left(T^{*}\right)\right)+\int_{t_{0}}^{t} p(s) \Delta s+\int_{t_{0}}^{t} f(s)\left[v(s)+\int_{t_{0}}^{s} g(s, \lambda) v(\lambda) \Delta \lambda\right] \Delta s\right] \\
& \leq \tilde{G}^{-1}\left[G\left(a\left(T^{*}\right)\right)+\int_{t_{0}}^{T^{*}} p(s) \Delta s+\int_{t_{0}}^{t} f(s)\left[v(s)+\int_{t_{0}}^{s} g(s, \lambda) v(\lambda) \Delta \lambda\right] \Delta s\right] .
\end{aligned}
$$

For brevity, let

$$
z(t)=\tilde{G}\left(a\left(T^{*}\right)\right)+\int_{t_{0}}^{T^{*}} p(s) \Delta s+\int_{t_{0}}^{t} f(s)\left[v(s)+\int_{t_{0}}^{s} g(s, \lambda) v(\lambda) \Delta \lambda\right] \Delta s,
$$

and hence

$$
z\left(t_{0}\right)=\tilde{G}\left(a\left(T^{*}\right)\right)+\int_{t_{0}}^{T^{*}} p(s) \Delta s
$$

Then

$$
v(t) \leq \tilde{G}^{-1}(z(t)), \quad t \in\left[t_{0}, T^{*}\right] \cap \mathbb{T} .
$$

Using Lemma 1.2, (2.34), and (2.33), we have

$$
\begin{aligned}
z^{\Delta}(t) & =f(t)\left[v(t)+\int_{t_{0}}^{t} g(s) v(s) \Delta s\right] \\
& \leq f(t)\left[1+\int_{t_{0}}^{t} g(t, s) \Delta s\right] \tilde{G}^{-1}(z(t)) .
\end{aligned}
$$

From (2.35) we have

$$
\frac{z^{\Delta}(t)}{\tilde{G}^{-1}(z(t))} \leq f(t)\left[1+\int_{t_{0}}^{t} g(t, s) \Delta s\right]
$$

Integrating both sides of (2.36) from $t_{0}$ to $t$ yields

$$
\tilde{H}(z(t))-\tilde{H}(z(t)) \leq \int_{t_{0}}^{t} f(s)\left[1+\int_{t_{0}}^{s} g(s, \lambda) \Delta \lambda\right] \Delta s,
$$


which implies

$$
\begin{aligned}
z(t) \leq & \tilde{H}^{-1}\left\{\tilde{H}\left[\left(G\left(a\left(T^{*}\right)\right)\right)+\int_{t_{0}}^{T^{*}} p(s) \Delta s\right]\right. \\
& \left.+\int_{t_{0}}^{t} f(s)\left[1+\int_{t_{0}}^{s} g(s, \lambda) \Delta \lambda\right] \Delta s\right\} .
\end{aligned}
$$

From (2.27), (2.34), and (2.37) we obtain

$$
\begin{aligned}
u(t) \leq & \omega_{1}^{-1}\left\{\tilde { G } ^ { - 1 } \left[\tilde { H } ^ { - 1 } \left\{\tilde{H}\left[G\left(a\left(T^{*}\right)\right)+\int_{t_{0}}^{T^{*}} p(s) \Delta s\right]\right.\right.\right. \\
& \left.\left.\left.+\int_{t_{0}}^{T^{*}} f(s)\left[1+\int_{t_{0}}^{s} g(s, \lambda) \Delta \lambda\right] \Delta s\right\}\right]\right\} .
\end{aligned}
$$

Taking $t=T^{*}$ in (2.38) yields

$$
\begin{aligned}
u\left(T^{*}\right) \leq & \omega_{1}^{-1}\left\{\tilde { G } ^ { - 1 } \left[\tilde { H } ^ { - 1 } \left\{\tilde{H}\left[G\left(a\left(T^{*}\right)\right)+\int_{t_{0}}^{T^{*}} p(s) \Delta s\right]\right.\right.\right. \\
& \left.\left.\left.+\int_{t_{0}}^{T^{*}} f(s)\left[1+\int_{t_{0}}^{s} g(s, \lambda) \Delta \lambda\right] \Delta s\right\}\right]\right\} .
\end{aligned}
$$

Since $T^{*} \in \mathbb{T}_{0}$ is chosen arbitrarily, we obtain the required result after substituting $T^{*}$ with $t$ into (2.39). This completes the proof.

Remark 2.7 It is interesting to note that, as a particular case, if we put $\omega_{1}(u)=u, \omega_{2}(u)=1$, $\tau(t)=t$, and $a(t)=u_{0}$ (any constant) in Theorem 2.6, then the inequality given in Theorem 2.6 reduces to the inequality given in [31, Thm. 3.1].

Remark 2.8 In addition to the assumptions in Remark 2.7, if we put $p(t)=0$ and $g(t, s)=$ $g(s)$ in Theorem 2.6, then we get $[9, \mathrm{Thm} .1]$ as a particular case.

Theorem 2.6 unifies some known continuous and discrete inequalities in the literature as shown in the following remarks:

Remark 2.9 In addition to the assumptions in Remark 2.7, when $p(t)=0$ in Theorem 2.6, if $\mathbb{T}=\mathbb{R}$, then we can easily obtain the continuous version proved by Pachpatte $[8$, Thm. 2.1 $\left(a_{1}\right)$ ]. If $\mathbb{T}=\mathbb{Z}$, then we can also get the discrete version proved by Pachpatte $\left[8, \mathrm{Thm} .2 .3\left(c_{1}\right)\right]$.

Remark 2.10 It is interesting to note that if, in addition to the assumptions in Remark 2.7, we take $g(t, s)=g(s)$ in Theorem 2.6 and $\mathbb{T}=\mathbb{R}$, then we get the continuous version established by Pachpatte [8, Thm. 1.7.2(i)]. Furthermore, if $\mathbb{T}=\mathbb{Z}$, then we can also get the discrete version inequality established by Pachpatte [8, Thm. 1.8.7].

Theorem 2.11 Let $u, a, \tau, \omega(u), h, g$ be defined as in Theorem 2.1. If $t \in \mathbb{T}_{0}$ and $u$ satisfies the retarded dynamic inequality

$$
u^{p}(t) \leq a(t)+\int_{t_{0}}^{t} h(t, s) \omega(u(\tau(s)))\left[\omega(u(\tau(s)))+\int_{t_{0}}^{s} g(s, \lambda) \omega(u(\tau(\lambda))) \Delta \lambda\right] \Delta s
$$


with the initial condition

$$
\left\{\begin{array}{l}
u(t)=\phi(t) \quad \text { if } t \in\left[\alpha, t_{0}\right] \cap \mathbb{T}, \\
\phi(\tau(t)) \leq a^{\frac{1}{p}}(t) \quad \text { if } \tau(t) \leq t_{0}, t \in \mathbb{T}_{0},
\end{array}\right.
$$

then

$$
\begin{aligned}
u(t) \leq & \left\{\Omega ^ { - 1 } \left[\Upsilon^{-1}\{\Upsilon(\Omega(v(a(t))))\right.\right. \\
& \left.\left.\left.+\int_{t_{0}}^{t} h(t, s)\left[1+\int_{t_{0}}^{s} g(s, \lambda) \Delta \lambda\right] \Delta s\right\}\right]\right\}^{\frac{1}{p}}
\end{aligned}
$$

with

$$
\Upsilon(\Omega(v(a(t))))+\int_{t_{0}}^{t} h(t, s)\left[1+\int_{t_{0}}^{s} g(s, \lambda) \Delta \lambda\right] \Delta s \in \operatorname{Dom}\left(\Upsilon^{-1}\right),
$$

where $\Omega$ and $\Upsilon$ are increasing bijective functions defined by

$$
\begin{aligned}
& (\Omega \circ v)^{\Delta}(t)=\frac{v^{\Delta}(t)}{\omega\left(v^{\frac{1}{p}}(t)\right)}, \\
& (\Upsilon \circ z)^{\Delta}(t)=\frac{z^{\Delta}(t)}{\omega\left(\left[\Omega^{-1}(z(t))\right]^{\frac{1}{p}}\right)} .
\end{aligned}
$$

Proof Fixing an arbitrary number $T^{*} \in \mathbb{T}_{0}$, for $t \in\left[t_{0}, T^{*}\right] \cap \mathbb{T}$, we define the function

$$
\begin{aligned}
v(t)= & a\left(T^{*}\right)+\int_{t_{0}}^{t} h(t, s) \omega(u(\tau(s)))[\omega(u(\tau(s))) \\
& \left.+\int_{t_{0}}^{s} \omega(u(\tau(\lambda))) \Delta \lambda\right] \Delta s .
\end{aligned}
$$

Clearly, $v$ is a nonnegative nondecreasing function with $v\left(t_{0}=a\left(T^{*}\right)\right)$, and we have

$$
u(t) \leq v^{\frac{1}{p}}(t), \quad t \in\left[t_{0}, T^{*}\right] \cap \mathbb{T} .
$$

Therefore, for $t \in\left[t_{0}, T^{*}\right] \cap \mathbb{T}$, if $\tau(t) \geq t_{0}$, then $\tau(t) \in\left[t_{0}, T^{*}\right] \cap \mathbb{T}$, and from (2.46) we obtain

$$
u(\tau(t)) \leq v^{\frac{1}{p}}(\tau(t)) \leq v^{\frac{1}{p}}(t) .
$$

On the other hand, if $\tau(t) \leq t_{0}$, then, using the initial condition (2.41), we get

$$
u(\tau(t))=\phi(\tau(t)) \leq a^{\frac{1}{p}}(t) \leq v^{\frac{1}{p}}(t) .
$$

So, combining (2.47) and (2.48), we have

$$
u(\tau(t)) \leq v^{\frac{1}{p}}(t) .
$$


Thus from (2.45), Lemma 1.2, and (2.49) we have

$$
\begin{aligned}
v^{\Delta}(t)= & \int_{t_{0}}^{t} h^{\Delta t}(t, s) \omega(u(\tau(s)))\left[\omega(u(\tau(s)))+\int_{t}^{s} g(t, s) \omega(u(\tau(\lambda))) \Delta \lambda\right] \Delta s \\
& +h(\sigma(t), t) \omega(u(\tau(t)))\left[\omega(u(\tau(t)))+\int_{t}^{t} g(t, s) \omega(u(\tau(s))) \Delta s\right] \\
\leq & \left\{\int_{t_{0}}^{t} h^{\Delta t}(t, s)\left[\omega\left(v^{\frac{1}{p}}(s)\right)+\int_{t}^{s} g(t, s) \omega\left(v^{\frac{1}{p}}(\lambda)\right) \Delta \lambda\right] \Delta s\right. \\
& \left.+h(\sigma(t), t)\left[\omega\left(v^{\frac{1}{p}}(t)\right)+\int_{t}^{t} g(t, s) \omega\left(v^{\frac{1}{p}}(s)\right) \Delta s\right]\right\} \omega\left(v^{\frac{1}{p}}(t)\right),
\end{aligned}
$$

which implies

$$
\begin{aligned}
\frac{v^{\Delta}(t)}{\omega\left(v^{\frac{1}{p}}(t)\right)} \leq & \int_{t_{0}}^{t} h^{\Delta_{t}}(t, s)\left[\omega\left(v^{\frac{1}{p}}(t)\right)+\int_{t}^{s} g(t, s) \omega\left(v^{\frac{1}{p}}(\lambda)\right) \Delta \lambda\right] \Delta s \\
& +h(\sigma(t), t)\left[\omega\left(v^{\frac{1}{p}}(t)\right)+\int_{t}^{t} g(t, s) \omega\left(v^{\frac{1}{p}}(s)\right) \Delta s\right] \\
= & \left\{\int_{t_{0}}^{t} h(\sigma(t), t)\left[\omega\left(v^{\frac{1}{p}}(t)\right)+\int_{t}^{s} g(s, \lambda) \omega\left(v^{\frac{1}{p}}(\lambda)\right) \Delta \lambda\right] \Delta s\right\}^{\Delta_{t}} .
\end{aligned}
$$

Integrating both sides of (2.51) from $t_{0}$ to $t$ yields

$$
\Omega(v(t))-\Omega\left(v\left(t_{0}\right)\right) \leq \int_{t_{0}}^{t} h(t, s)\left[\omega\left(v^{\frac{1}{p}}(s)\right)+\int_{t_{0}}^{s} g(s, \lambda) \omega\left(v^{\frac{1}{p}}(\lambda)\right) \Delta \lambda\right] \Delta s .
$$

Since $\Omega$ is increasing, (2.52) implies

$$
\begin{aligned}
v(t) \leq & \Omega^{-1}\left[\Omega\left(v\left(a\left(T^{*}\right)\right)\right)+\int_{t_{0}}^{t} h(t, s)\left[\omega\left(v^{\frac{1}{p}}(s)\right)\right.\right. \\
& \left.\left.+\int_{t_{0}}^{s} g(s, \lambda) \omega\left(v^{\frac{1}{p}}(\lambda)\right) \Delta \lambda\right] \Delta s\right] .
\end{aligned}
$$

For brevity, let

$$
z(t)=\Omega\left(v\left(a\left(T^{*}\right)\right)\right)+\int_{t_{0}}^{t} h(t, s)\left[\omega\left(v^{\frac{1}{p}}(s)\right)+\int_{t_{0}}^{s} g(s, \lambda) \omega\left(v^{\frac{1}{p}}(\lambda)\right) \Delta \lambda\right] \Delta s,
$$

and hence $z\left(t_{0}\right)=\Omega\left(v\left(T^{*}\right)\right)$.

Then

$$
v(t) \leq \Omega^{-1}(z(t)), \quad t \in\left[t_{0}, T^{*}\right] \cap \mathbb{T} .
$$

By Lemma 1.2, (2.54), and (2.55) we get

$$
\begin{aligned}
z^{\Delta}(t)= & \int_{t_{0}}^{t} h^{\Delta_{t}}(t, s)\left[\omega\left(v^{\frac{1}{p}}(s)\right)+\int_{t_{0}}^{t} g(s, \lambda) \omega\left(v^{\frac{1}{p}}(\lambda)\right) \Delta \lambda\right] \Delta s \\
& +h(\sigma(t), t)\left[\omega\left(v^{\frac{1}{p}}(t)\right)+\int_{t_{0}}^{t} g(t, s) \omega\left(v^{\frac{1}{p}}(s)\right) \Delta s\right]
\end{aligned}
$$




$$
\begin{aligned}
\leq & \left\{\int_{t_{0}}^{t} h^{\Delta t}(t, s)\left[1+\int_{t_{0}}^{t} g(s, \lambda) \Delta \lambda\right] \Delta s+h(\sigma(t), s)\left[1+\int_{t_{0}}^{t} g(t, s) \Delta s\right]\right\} \\
& \times \omega\left(\left[\Omega^{-1}(z(t))\right]^{\frac{1}{p}}\right)
\end{aligned}
$$

which implies

$$
\frac{z^{\Delta}(t)}{\omega\left(\left[\Omega^{-1}(z(t))\right]^{\frac{1}{p}}\right)} \leq\left\{\int_{t_{0}}^{t} h(t, s)\left[1+\int_{t_{0}}^{s} g(s, \lambda) \Delta \lambda\right] \Delta s\right\}^{\Delta_{t}} .
$$

Integrating both sides of (2.56) from $t_{0}$ to $t$ yields

$$
\Upsilon(z(t))-\Upsilon\left(z\left(t_{0}\right)\right) \leq \int_{t_{0}}^{t} h(t, s)\left[1+\int_{t_{0}}^{s} g(s, \lambda) \Delta \lambda\right] \Delta s .
$$

Since $\Upsilon$ is increasing, (2.57) implies

$$
z(t) \leq \Upsilon^{-1}\left[\Upsilon\left(\Omega\left(v\left(a\left(T^{*}\right)\right)\right)\right)+\int_{t_{0}}^{t} h(t, s)\left[1+\int_{t_{0}}^{s} g(s, \lambda) \Delta \lambda\right] \Delta s\right] .
$$

Combining (2.46), (2.55), and (2.58), we obtain

$$
\begin{aligned}
u(t) \leq & {\left[\Omega ^ { - 1 } \left\{\Upsilon ^ { - 1 } \left[\Upsilon\left(\Omega\left(v\left(a\left(T^{*}\right)\right)\right)\right)\right.\right.\right.} \\
& \left.\left.\left.+\int_{t_{0}}^{t} h(t, s)\left[1+\int_{t_{0}}^{s} g(s, \lambda) \Delta \lambda\right] \Delta s\right]\right\}\right]^{\frac{1}{p}} .
\end{aligned}
$$

Taking $t=T^{*}$ in (2.38) yields

$$
\begin{aligned}
u\left(T^{*}\right) \leq & {\left[\Omega ^ { - 1 } \left\{\Upsilon ^ { - 1 } \left[\Upsilon\left(\Omega\left(v\left(a\left(T^{*}\right)\right)\right)\right)\right.\right.\right.} \\
& \left.\left.\left.+\int_{t_{0}}^{T^{*}} h(t, s)\left[1+\int_{t_{0}}^{s} g(s, \lambda) \Delta \lambda\right] \Delta s\right]\right\}\right]^{\frac{1}{p}} .
\end{aligned}
$$

Since $T^{*} \in \mathbb{T}_{0}$ is chosen arbitrarily, we obtain the required inequality after substituting $T^{*}$ with $t$ into (2.60). This completes the proof.

Remark 2.12 If we take $h(t, s)=f(s), g(s, \lambda)=g(\lambda), p=1, a(t)=u_{0}$ (any constant), and $\mathbb{T}=\mathbb{R}$ in Theorem 2.11, then the inequality given in Theorem 2.11 reduces to the inequality given in [10, Thm. 2.1].

Theorem 2.13 Letu, $a, \omega_{1}, \omega_{2}, \tau$ be defined as in Theorem 2.1, and let $f, g \in C_{\mathrm{rd}}\left(\mathbb{T}_{0}, \mathbb{R}^{+}\right)$. If $u$ satisfies the inequality

$$
u(t) \leq a(t)+\int_{0}^{t} f(s) \psi_{1}(u(\tau(s)))\left[u^{q}(\tau(s))+\int_{0}^{s} g(\lambda) \psi_{2}(u(\tau(\lambda))) \Delta \lambda\right]^{p} \Delta s
$$

with the initial condition

$$
\left\{\begin{array}{l}
u(t)=\phi(t) \quad \text { if } t \in\left[\alpha, t_{0}\right] \cap \mathbb{T}, \\
\phi(\tau(t)) \leq a(t) \quad \text { if } \tau(t) \leq t_{0}, t \in \mathbb{T}_{0}
\end{array}\right.
$$


where $p>0$ and $q \geq 1$ are constants such that $p+q>1$, then

$$
u(t) \leq \Psi_{1}^{-1}\left[\Psi_{2}^{-1}\left(\Psi_{2}\left(\Psi_{1}\left(u_{0}^{q}\right)+\int_{0}^{t} g(s) \Delta s\right)+\int_{0}^{t} f(s) \Delta s\right)\right]
$$

with

$$
\Psi_{2}\left(\Psi_{1}\left(u_{0}^{q}\right)+\int_{0}^{t} g(s) \Delta s\right)+\int_{0}^{t} f(s) \Delta s \in \operatorname{Dom} \Psi_{2}^{-1}
$$

where

$$
\begin{aligned}
& \left(\Psi_{1} \circ Y\right)^{\Delta}(t)=\frac{Y^{\Delta}(t)}{\psi_{2}(Y(t))}, \\
& \left(\Psi_{2} \circ Y_{1}\right)^{\Delta}(t)=\frac{\psi_{2}\left(\Psi_{1}^{-1}\left(Y_{1}(t)\right)\right) Y_{1}^{\Delta}(t)}{\psi_{1}\left(\Psi_{1}^{-1}\left(Y_{1}(t)\right)\right)\left[\Psi_{1}^{-1}\left(Y_{1}(\sigma(t))\right)\right]^{\frac{q+p q-1}{q}}} .
\end{aligned}
$$

Proof Fixing an arbitrary number $T^{*} \in \mathbb{T}_{0}$, for $t \in\left[t_{0}, T^{*}\right] \cap \mathbb{T}$, we define the function

$$
v(t)=a\left(T^{*}\right)+\int_{t_{0}}^{t} f(s) \omega_{1}(u(\tau(s)))\left[u^{q}(\tau(s))+\int_{0}^{s} g(\lambda) \omega_{2}(u(\tau(\lambda))) \Delta \lambda\right]^{p} \Delta s,
$$

which is a positive nondecreasing function with $v\left(t_{0}\right)=a\left(T^{*}\right)$. Then we have

$$
u(t) \leq v(t), \quad t \in\left[t_{0}, T^{*}\right] \cap \mathbb{T} .
$$

Therefore, for $t \in\left[t_{0}, T^{*}\right] \cap \mathbb{T}$, if $\tau(t) \geq t_{0}$, then $\tau(t) \in\left[t_{0}, T^{*}\right] \cap \mathbb{T}$, and from (2.46) we obtain

$$
u(\tau(t)) \leq v(t)
$$

On the other hand, if $\tau(t) \leq t_{0}$, then, using the initial condition (2.62), we get

$$
u(\tau(t))=\phi(\tau(t)) \leq a(t) \leq v(t)
$$

So, combining (2.68) and (2.69), we have

$$
u(\tau(t)) \leq v(t), \quad t \in\left[t_{0}, T^{*}\right] \cap \mathbb{T} .
$$

Thus, by Lemma 1.2 from (2.66) and (2.70) we get

$$
v^{\Delta}(t)=f(t) \omega_{1}(v(t)) Y^{p}(t)
$$

where $Y(t)=v^{q}(t)+\int_{t_{0}}^{t} g(s) \omega_{2}(v(s)) \Delta s$ is nondecreasing. Hence $Y\left(t_{0}\right)=v^{q}\left(t_{0}\right)=a^{q}\left(T^{*}\right)$ and $\nu^{q}(t) \leq Y(t)$, but $q \geq 1$, and thus

$$
\begin{aligned}
& v(t) \leq Y^{\frac{1}{q}}(t), \\
& v^{\sigma}(t) \leq Y^{\frac{1}{q}}(\sigma(t))
\end{aligned}
$$

for all $t \in\left[t_{0}, T^{*}\right] \cap \mathbb{T}$. 
Since $v$ is nondecreasing, from $Y(t)$, by Lemma 1.7 and Lemma 1.2 we see that

$$
Y^{\Delta}(t) \leq q\left[v^{\sigma}(t)\right]^{q-1} v^{\Delta}(t)+g(t) \omega_{2}(v(t))
$$

From (2.73), using (2.71), (2.72), and the inequality $Y(t) \leq Y^{\sigma}(t)$, we get

$$
\begin{aligned}
Y^{\Delta}(t) & \leq q\left[Y^{\sigma}(t)\right]^{\frac{q-1}{q}} f(t) \omega_{1}(Y(t)) Y^{p}(t)+g(t) \omega_{2}(Y(t)) \\
& \leq q f(t) \omega_{1}(Y(t))\left[Y^{\sigma}(t)\right]^{\frac{q-1}{q}}\left[Y^{\sigma}(t)\right]^{p}+g(t) \omega_{2}(Y(t)) \\
& =q f(t) \omega_{1}(Y(t))\left[Y^{\sigma}(t)\right]^{\frac{q+p q-1}{q}}+g(t) \omega_{2}(Y(t)),
\end{aligned}
$$

$t \in\left[t_{0}, T^{*}\right] \cap \mathbb{T}$.

Since $\omega_{2}(Y(t))>0$ for $t>0$, from (2.74) we get

$$
\frac{Y^{\Delta}(t)}{\omega_{2}(Y(t))} \leq q f(t) \frac{\omega_{1}(Y(t))\left[Y^{\sigma}(t)\right]^{\frac{q+p q-1}{q}}}{\omega_{2}(Y(t))}+g(t)
$$

Taking $t=s$ in the last inequality, integrating both sides from $t_{0}$ to $t$, and using (2.64), we have

$$
\Psi_{1}(Y(t)) \leq \Psi_{1}\left(a^{q}\left(T^{*}\right)\right)+\int_{t_{0}}^{t} q f(s) \frac{\omega_{1}(Y(s))\left[Y^{\sigma}(s)\right]^{\frac{q+p q-1}{q}}}{\omega_{2}(Y(s))} \Delta s+\int_{t_{0}}^{t} g(s) \Delta s
$$

where $\Psi_{1}$ is defined by (2.64). From this inequality we have

$$
\begin{aligned}
\Psi_{1}(Y(t)) \leq & \Psi_{1}\left(a^{q}\left(T^{*}\right)\right)+\int_{t_{0}}^{T^{*}} g(s) \Delta s \\
& +\int_{t_{0}}^{t} q f(s) \frac{\omega_{1}(Y(s))\left[Y^{\sigma}(s)\right]^{\frac{q+p q-1}{q}}}{\omega_{2}(Y(s))} \Delta s .
\end{aligned}
$$

Let $Y_{1}(t)$ denote the function on the right-hand side of this inequality. Then

$$
\begin{aligned}
Y_{1}(t)= & \Psi_{1}\left(a^{q}\left(T^{*}\right)\right)+\int_{t_{0}}^{T^{*}} g(s) \Delta s \\
& +\int_{t_{0}}^{t} q f(s) \frac{\omega_{1}(Y(s)) Y^{\frac{q+p q-1}{q}}(s)}{\omega_{2}(Y(s))} \Delta s,
\end{aligned}
$$

which is a positive nondecreasing function with $Y_{1}\left(T^{*}\right)=\Psi_{2}\left(a^{q}\left(T^{*}\right)\right)+\int_{t_{0}}^{T^{*}} g(s) \Delta s$, and

$$
\begin{aligned}
& Y(t) \leq \Psi_{1}^{-1}\left(Y_{1}(t)\right), \\
& Y^{\sigma}(t) \leq \Psi_{1}^{-1}\left(Y_{1}(\sigma(t))\right) .
\end{aligned}
$$


From (2.75), (2.76), and Lemma 1.2 we obtain

$$
\begin{aligned}
Y_{1}^{\Delta}(t) & \leq q f(t) \frac{\omega_{1}(Y(t)) Y^{\frac{q+p q-1}{q}}(t)}{\omega_{2}(Y(t))} \\
& \leq q f(t) \frac{\omega_{1}\left(\Psi_{1}^{-1}\left(Y_{1}(t)\right)\right)\left[\Psi_{1}^{-1}\left(Y_{1}(\sigma(t))\right)\right]^{\frac{q+p q-1}{q}}(t)}{\omega_{2}\left(\Psi_{1}^{-1}\left(Y_{1}(\sigma(t))\right)\right)} .
\end{aligned}
$$

From this inequality, by the definition of $\Psi_{2}$ in (2.65), letting $t=T^{*}$, we have

$$
\Psi_{2}\left(Y_{1}\left(T^{*}\right)\right) \leq \Psi_{2}\left(\Psi_{1}\left(a^{q}\left(T^{*}\right)\right)+\int_{t_{0}}^{T^{*}} g(s) \Delta s\right)+\int_{t_{0}}^{T^{*}} q f(s) \Delta s .
$$

Since $T^{*} \in \mathbb{T}_{0}$ is chosen, from (2.70), (2.72), (2.76), and (2.77) we get the required inequality in (2.63). This completes the proof.

Remark 2.14 By taking $\mathbb{T}=\mathbb{R}$ and $a(t)=u_{0}$ (any constant) in Theorem 2.13 it is easy to observe that the inequality obtained in Theorem 2.13 reduces to the inequality obtained by Abdeldaim and El-Deeb in [13, Thm. 2.5].

Remark 2.15 If we put $\omega_{1}(u)=\omega_{2}(u)=u, a(t)=u_{0}$ (any constant), and $\mathbb{T}=\mathbb{R}$ in Theorem 2.13, then we get the inequality obtained by Abdeldaim and El-Deeb in [13, Thm. 2.3].

Remark 2.16 If we take $q=1, a(t)=u_{0}$ (any constant), and $\mathbb{T}=\mathbb{R}$ in Theorem 2.13 , then the inequality given in Theorem 2.13 reduces to the inequality given in [40, Thm. 4].

\section{Applications}

In this section, we study the uniqueness and global existence of solutions for a class of nonlinear delay dynamic integral equations.

First, we consider the following delay dynamic equation on time scales:

$$
\left(u^{p}\right)^{\Delta}(t)=E\left(t, s, \omega(u(\tau(t))), \int_{t_{0}}^{t} F(t, s, \omega(u(\tau(s)))) \Delta s\right)
$$

with the initial condition

$$
\left\{\begin{array}{l}
u\left(t_{0}\right)=c, \\
u(t)=\phi(t) \quad \text { if } t \in\left[\alpha, t_{0}\right] \cap \mathbb{T} ; \\
\phi(\tau(t)) \leq c^{\frac{1}{p}}, \quad t \in \mathbb{T}_{0}, \text { if } \tau(t) \leq t_{0},
\end{array}\right.
$$

where $u \in C_{\mathrm{rd}}\left(\mathbb{T}_{0}, \mathbb{R}^{+}\right), \phi \in C_{\mathrm{rd}}\left(\left[\alpha, t_{0}\right] \cap \mathbb{T}\right), c \neq 0$ is a constant, $\alpha, \tau$ are defined as in Theorem 2.11, and $E: \mathbb{T}_{0}^{2} \times \mathbb{R}^{2} \longrightarrow \mathbb{R}$ and $F: \mathbb{T}_{0}^{2} \times \mathbb{R} \longrightarrow \mathbb{R}$ are continuous functions.

Theorem 3.1 Assume that $u$ is a solution of the delay dynamic equation (3.1) with initial condition (3.2) and suppose that

$$
\begin{aligned}
& E(t, s, \omega(u), v) \leq h(t, s) \omega(u)[\omega(u)+v], \\
& F(t, s, \omega(u)) \leq g(t, s) \omega(u),
\end{aligned}
$$


where $h, g, \omega$ are defined as in Theorem 2.11. Then we get

$$
\begin{aligned}
u(t) \leq & \left\{\hat { \Omega } ^ { - 1 } \left[\hat{\Upsilon}^{-1}\{\hat{\Upsilon}(\hat{\Omega}(v(c)))\right.\right. \\
& \left.\left.\left.+\int_{t_{0}}^{t} h(t, s)\left[1+\int_{t_{0}}^{s} g(s, \lambda) \Delta \lambda\right] \Delta s\right\}\right]\right\}^{\frac{1}{p}}
\end{aligned}
$$

with

$$
\hat{\Upsilon}(\hat{\Omega}(v(c)))+\int_{t_{0}}^{t} h(t, s)\left[1+\int_{t_{0}}^{s} g(s, \lambda) \Delta \lambda\right] \Delta s \in \operatorname{Dom}\left(\hat{\Upsilon}^{-1}\right),
$$

where $\hat{\Omega}, \hat{\Upsilon}$ are increasing bijective function, and

$$
\begin{aligned}
& (\hat{\Omega} \circ v)^{\Delta}(t)=\frac{v^{\Delta}(t)}{\omega\left(v^{\frac{1}{p}}(t)\right)}, \\
& (\hat{\Upsilon} \circ z)^{\Delta}(t)=\frac{z^{\Delta}(t)}{\omega\left(\left[\hat{\Omega}^{-1}(z(t))\right]^{\frac{1}{p}}\right)} .
\end{aligned}
$$

Proof Clearly, the solution $u$ of the delay dynamic equation (3.1) with initial condition (3.2) satisfies the equivalent delay dynamic integral equation

$$
u^{p}(t)=C+\int_{t_{0}}^{t} E\left(t, s, \omega(u(\tau(s))), \int_{t_{0}}^{s} F(t, \xi, \omega(u(\tau(\xi)))) \Delta \xi\right) \Delta s
$$

with initial condition (3.2). Using hypotheses (3.3) and (3.4), we get

$$
\begin{aligned}
\left|u^{p}(t)\right|= & \left|c+\int_{t_{0}}^{t} E\left(t, s, \omega(u(\tau(s))), \int_{t_{0}}^{s} F(t, \xi, \omega(u(\tau(\xi)))) \Delta \xi\right) \Delta s\right| \\
\leq & |c|+\left|\int_{t_{0}}^{t} E\left(t, s, \omega(u(\tau(s))), \int_{t_{0}}^{s} F(t, \xi, \omega(u(\tau(\xi)))) \Delta \xi\right) \Delta s\right| \\
\leq & |c|+\int_{t_{0}}^{t}\left|E\left(t, s, \omega(u(\tau(s))), \int_{t_{0}}^{s} F(t, \xi, \omega(u(\tau(\xi)))) \Delta \xi\right)\right| \Delta s \\
\leq & |c|+\int_{t_{0}}^{t} h(t, s) \omega(|u(\tau(s))|)[\omega(|u(\tau(s))|) \\
& \left.+\int_{t_{0}}^{s} g(s, \lambda) \omega(|u(\tau(\lambda))|) \Delta \lambda\right] \Delta s
\end{aligned}
$$

with initial condition (3.2). Now a suitable application of Theorem 2.11 to (3.6) yields

$$
\begin{aligned}
u(t) \leq & \left\{\hat { \Omega } ^ { - 1 } \left[\hat{\Upsilon}^{-1}\{\hat{\Upsilon}(\Omega(v(c)))\right.\right. \\
& \left.\left.\left.+\int_{t_{0}}^{t} h(t, s)\left[1+\int_{t_{0}}^{s} g(s, \lambda) \Delta \lambda\right] \Delta s\right\}\right]\right\}^{\frac{1}{p}} .
\end{aligned}
$$

This is the required estimate in (3.5). This completes the proof. 
Theorem 3.2 Assume that

$$
\begin{aligned}
& \left|E\left(t, s, \omega\left(u_{1}\right), \gamma_{1}\right)-E\left(t, s, \omega\left(u_{2}\right), \gamma_{2}\right)\right| \\
& \quad \leq h(t, s)\left|\omega\left(u_{1}\right)-\omega\left(u_{2}\right)\right|\left(\left|\omega\left(u_{1}\right)-\omega\left(u_{2}\right)\right|+\left|\gamma_{1}-\gamma_{2}\right|\right), \\
& \left|F\left(t, s, \omega\left(u_{1}\right)\right)-F\left(t, s, \omega\left(u_{2}\right)\right)\right| \leq g(t, s)\left|\omega\left(u_{1}\right)-\omega\left(u_{2}\right)\right|, \\
& \left|\omega\left(u_{1}\right)-\omega\left(u_{2}\right)\right| \leq\left|u_{1}^{p}-u_{2}^{p}\right|,
\end{aligned}
$$

where $h, g, \omega_{1}$, and $\omega_{2}$ are defined as in Theorem 2.11. Then the delay dynamic equation (3.1) with initial condition (3.2) has at most one solution.

Proof Let $u_{1}(t)$ and $u_{2}(t)$ be two solutions of (3.1) with initial condition (3.2). Then we have

$$
\begin{aligned}
u_{1}^{p}(t)-u_{2}^{p}(t)= & \int_{t_{0}}^{t} E\left(t, s, \omega\left(u_{1}(\tau(s))\right), \int_{t_{0}}^{s} F\left(t, \xi, \omega\left(u_{1}(\tau(\xi))\right)\right) \Delta \xi\right) \Delta s \\
& -\int_{t_{0}}^{t} E\left(t, s, \omega\left(u_{2}(\tau(s))\right), \int_{t_{0}}^{s} F\left(t, \xi, \omega\left(u_{2}(\tau(\xi))\right)\right) \Delta \xi\right) \Delta s .
\end{aligned}
$$

Thus from (3.8), using hypotheses (3.7), we get

$$
\begin{aligned}
\left|u_{1}^{p}(t)-u_{2}^{p}(t)\right| \leq & \int_{t_{0}}^{t} h(t, s)\left|\omega\left(u_{1}(\tau)\right)-\omega\left(u_{2}(\tau)\right)\right|\left[\left|\omega\left(u_{1}(\tau)\right)-\omega\left(u_{2}(\tau)\right)\right|\right. \\
& \left.+\int_{t_{0}}^{s} g(s, \lambda)\left|\omega\left(u_{1}(\lambda)\right)-\omega\left(u_{2}(\lambda)\right)\right| \Delta \lambda\right] \Delta s .
\end{aligned}
$$

Applying Theorem 2.11, we have $\left|u_{1}(t)-u_{2}(t)\right| \equiv 0$. Therefore $u_{1}(t)=u_{2}(t)$. Then the delay dynamic equation (3.1) with initial condition (3.2) has at most one solution. This completes the proof.

\section{Acknowledgements}

The authors wish to express their sincere appreciation to the editor and the anonymous referees for their valuable comments and suggestions.

\section{Funding}

This research was partially supported by the National Natural Science Foundation of China (No. 11501342) and the Natural Science Foundation for Young Scientists of Shanxi Province, China (No. 201701D221007).

\section{Competing interests}

The authors declare that they have no competing interests.

\section{Authors' contributions}

All authors contributed equally. All authors read and approved the final manuscript.

\section{Author details}

${ }^{1}$ Department of Mathematics, Faculty of Science, Al-Azhar University, Cairo, Egypt. ${ }^{2}$ College of Science \& Technology, Ningbo University, Ningbo, People's Republic of China. ${ }^{3}$ Community College, Shaqra University, Al-Dawadmi, Saudi Arabia. ${ }^{4}$ Mathematics Department, Faculty of Science, Port Said University, Port Said, Egypt. ${ }^{5}$ School of Mathematics and Computer Science, Shanxi Normal University, Linfen, People's Republic of China. ${ }^{6}$ College of Mathematics and System Science, Shandong University of Science and Technology, Qingdao, People's Republic of China. ${ }^{7}$ Nonlinear Analysis and Applied Mathematics (NAAM) Research Group, Department of Mathematics, Faculty of Science, King Abdulaziz University, Jeddah, Saudi Arabia.

\section{Publisher's Note}

Springer Nature remains neutral with regard to jurisdictional claims in published maps and institutional affiliations. 


\section{References}

1. Gronwall, T.H.: Note on the derivatives with respect to a parameter of the solutions of a system of differential equations. Ann. Math. (2) 20(4), 292-296 (1919)

2. Bellman, R.: The stability of solutions of linear differential equations. Duke Math. J. 10, 643-647 (1943)

3. Pachpatte, B.G.: On some fundamental integral inequalities and their discrete analogues. J. Inequal. Pure Appl. Math. 2(2), 1-13 (2001)

4. Rashid, M.H.M.: Explicit bounds on retarded Gronwall-Bellman inequality. Tamkang J. Math. 43(1), 99-108 (2012)

5. Ferreira, R.A.C., Torres, D.F.M.: Generalized retarded integral inequalities. Appl. Math. Lett. 22(6), 876-881 (2009)

6. Pachpatte, B.G.: Explicit bounds on certain integral inequalities. J. Math. Anal. Appl. 267(1), 48-61 (2002)

7. Abdeldaim, A., Yakout, M.: On some new integral inequalities of Gronwall-Bellman-Pachpatte type. Appl. Math. Comput. 217(20), 7887-7899 (2011)

8. Pachpatte, B.G.: Inequalities for Differential and Integral Equations. Mathematics in Science and Engineering, vol. 197. Academic Press, San Diego (1998)

9. Wong, F.-H., Yeh, C.-C., Hong, C.-H.: Gronwall inequalities on time scales. Math. Inequal. Appl. 9(1), 75-86 (2006)

10. Abdeldaim, A., El-Deeb, A.A.-M.: On some generalizations of certain retarded nonlinear integral inequalities with iterated integrals and an application in retarded differential equation. J. Egypt. Math. Soc. 23(3), 470-475 (2015)

11. Abdeldaim, A., El-Deeb, A.A.-M.: Some new retarded nonlinear integral inequalities with iterated integrals and their applications in retarded differential equations and integral equations. J. Fract. Calc. Appl. 5(suppl. 3S), Paper no. 9 (2014)

12. Abdeldaim, A., El-Deeb, A.A.-M.: On generalized of certain retarded nonlinear integral inequalities and its applications in retarded integro-differential equations. Appl. Math. Comput. 256, 375-380 (2015)

13. Abdeldaim, A., El-Deeb, A.A.-M.: On some new nonlinear retarded integral inequalities with iterated integrals and their applications in integro-differential equations. Br. J. Math. Comput. Sci. 5(4), 479-491 (2015)

14. El-Deeb, A.A., Ahmed, R.G.: On some generalizations of certain nonlinear retarded integral inequalities for Volterra-Fredholm integral equations and their applications in delay differential equations. J. Egypt. Math. Soc. 25(3), 279-285 (2017)

15. Li, W.N., Zhang, Q., Qiu, F.: Some nonlinear delay discrete inequalities and their applications. Demonstr. Math. 39(4), 771-782 (2006)

16. Pachpatte, B.G.: On some new inequalities related to a certain inequality arising in the theory of differential equations. J. Math. Anal. Appl. 251(2), 736-751 (2000)

17. Bihari, I.: A generalization of a lemma of Bellman and its application to uniqueness problems of differential equations. Acta Math. Acad. Sci. Hung. 7, 81-94 (1956)

18. El-Owaidy, H.M., Ragab, A.A., El-Deeb, A.A.-M., Abuelela, W.M.K.: On some new nonlinear integral inequalities of Gronwall-Bellman type. Kyungpook Math. J. 54(4), 555-575 (2014)

19. Agarwal, R.P., Deng, S., Zhang, W.: Generalization of a retarded Gronwall-like inequality and its applications. Appl. Math. Comput. 165(3), 599-612 (2005)

20. Bohner, M., Peterson, A.: Dynamic Equations on Time Scales: An Introduction with Applications. Birkhäuser Boston, Boston (2001)

21. Hilger, S.: Analysis on measure chains - a unified approach to continuous and discrete calculus. Results Math. 18(1-2), 18-56 (1990)

22. Bohner, M., Peterson, A.: Advances in Dynamic Equations on Time Scales. Birkhäuser Boston, Boston (2003)

23. Bohner, M., Erbe, L., Peterson, A.: Oscillation for nonlinear second order dynamic equations on a time scale. J. Math. Anal. Appl. 301(2), 491-507 (2005)

24. Tian, Y., El-Deeb, A.A., Meng, F.: Some nonlinear delay Volterra-Fredholm type dynamic integral inequalities on time scales. Discrete Dyn. Nat. Soc. 2018, Article ID 5841985 (2018)

25. El-Deeb, A.A., Cheung, W.-S.: A variety of dynamic inequalities on time scales with retardation. J. Nonlinear Sci. Appl. 11(10), 1185-1206 (2018)

26. El-Deeb, A.A., Elsennary, H.A., Nwaeze, E.R.: Generalized weighted Ostrowski, trapezoid and Grüss type inequalities on time scales. Fasc. Math. 60, 123-144 (2018)

27. Abdeldaim, A., El-Deeb, A.A., Agarwal, P., El-Sennary, H.A.: On some dynamic inequalities of Steffensen type on time scales. Math. Methods Appl. Sci. 41(12), 4737-4753 (2018)

28. El-Deeb, A.A., Elsennary, H.A., Cheung, W.-S.: Some reverse Hölder inequalities with Specht's ratio on time scales J. Nonlinear Sci. Appl. 11(4), 444-455 (2018)

29. Saker, S.H., El-Deeb, A.A., Rezk, H.M., Agarwal, R.P.: On Hilbert's inequality on time scales. Appl. Anal. Discrete Math 11(2), 399-423 (2017)

30. Akin-Bohner, E., Bohner, M., Akin, F.: Pachpatte inequalities on time scales. JIPAM. J. Inequal. Pure Appl. Math. 6(1), Article 6 (2005)

31. Li, W.N.: Some Pachpatte type inequalities on time scales. Comput. Math. Appl. 57(2), 275-282 (2009)

32. Li, W.N.: Some new dynamic inequalities on time scales. J. Math. Anal. Appl. 319(2), 802-814 (2006)

33. Li, W.N., Han, M.: Bounds for certain nonlinear dynamic inequalities on time scales. Discrete Dyn. Nat. Soc. 2009, Article ID 897087 (2009)

34. Saker, S.H.: Some nonlinear dynamic inequalities on time scales and applications. J. Math. Inequal. 4(4), 561-579 (2010)

35. Anderson, D.R.: Dynamic double integral inequalities in two independent variables on time scales. J. Math. Inequal. 2(2), 163-184 (2008)

36. Agarwal, R.P., Bohner, M., Peterson, A.: Inequalities on time scales: a survey. Math. Inequal. Appl. 4(4), $535-557$ (2001)

37. Feng, Q., Meng, F., Zheng, B.: Gronwall-Bellman type nonlinear delay integral inequalities on time scales. J. Math. Anal. Appl. 382(2), 772-784 (2011)

38. Yin, L., Luo, Q.-M., Qi, F.: Several integral inequalities on time scales. J. Math. Inequal. 6(3), 419-429 (2012)

39. Lipovan, O.: Integral inequalities for retarded Volterra equations. J. Math. Anal. Appl. 322(1), 349-358 (2006)

40. Wang, W.-S., Shen, C.-X.: On a generalized retarded integral inequality with two variables. J. Inequal. Appl. 2008 Article ID 518646 (2008) 